\title{
Sur une expression explicite de l'effet de rotation dans la fonction d'onde de vibration-rotation d'une molécule diatomique
}

\author{
H. Kobeissi
}

Faculté des Sciences, Université Libanaise et Le Groupe de Physique Moléculaire et Atomique au Conseil National de la Recherche Scientifique, Beyrouth, Liban.

(Reçu le 24 février 1981, accepté le 23 mars 1981)

Résumé. - L'étude de l'effet de rotation dans la fonction d'onde de vibration-rotation $\psi_{v J}$ est considérée. On démontre que cette fonction d'onde s'écrit, sans aucune restriction sur la nature du potentiel, sous la forme :

$$
\psi_{v J}=\psi_{v 0}+\sum_{i=1}^{\infty}[J(J+1)]^{i} \phi_{i} .
$$

Chaque fonction $\phi_{i}$ est la solution de l'équation radiale de Schroedinger de la vibration pure avec un second membre qui dépend de $\psi_{v 0}$ et des constantes rotationnelles $B_{v}, D_{v}, \ldots$ L'application numérique montre que les fonctions $\phi_{1}, \phi_{2}, \ldots$ décroissent comme $B_{v}, D_{v}, \ldots$

Abstract. - The effect of rotation on the vibration-rotational wavefunction $\psi_{v J}$ of a diatomic molecule is considered. It is shown that this function can be written, for any potential :

$$
\psi_{v J}=\psi_{v 0}+\sum_{i=1}^{\infty}[J(J+1)]^{i} \phi_{i}
$$

Each function $\phi_{i}$ is the solution of the purely vibrational radial Schroedinger equation with a second member depending on $\psi_{v 0}$ and the rotational constants $B_{v}, D_{v}, \ldots$ Numerical applications show that the functions $\phi_{1}, \phi_{2}, \ldots$ decrease in magnitude as do $B_{v}, D_{v}, \ldots$

1. Introduction. - Dans l'approximation de Born-Oppenheimer et pour un potentiel électronique $U(r)$ [où $r$ est la distance internucléaire], la fonction d'onde de vibration-rotation d'une molécule diatomique est la solution de l'équation radiale de Schroedinger [1] :

$\frac{\mathrm{d}^{2} \psi_{v J}(r)}{\mathrm{d} r^{2}}+\left[\frac{2 \mu}{\hbar^{2}}\left(E_{v J}-U(r)\right)-\frac{J(J+1)}{r^{2}}\right] \psi_{v J}(r)=0$

où $v$ et $J$ sont respectivement les nombres quantiques de vibration et de rotation; $\mu$ et $\hbar$ ont leurs significations habituelles.

Le potentiel $U(r)$ est le plus souvent donné sous une forme numérique $[2,3]$, et la fonction d'onde $\psi_{v J}$ est déterminée par des méthodes numériques [4] où l'effet de rotation figure implicitement dans la valeur numérique calculée de $\psi_{v}$.

Le but de ce travail est de dissocier dans $\psi_{v j}$, l'effet de la rotation de la fonction d'onde $\psi_{v 0}$ de la vibration pure.
2. Théorie. -2.1 Pour un niveau de vibration $v$, nous représentons dans la suite la fonction d'onde de vibration-rotation par la notation simplifiée $\psi_{\lambda}(x)$, où $\lambda=J(J+1)$ et $x=r-r_{\mathrm{e}}\left(r_{\mathrm{e}}\right.$ étant la valeur de $r$ à l'équilibre).

Nous savons déjà que la fonction d'onde $\psi_{\lambda}$ est la solution de l'équation radiale de Schroedinger :

$$
\psi_{\lambda}^{\prime \prime}(x)=f_{\lambda}(x) \psi_{\lambda}(x)
$$

ou de l'équation intégrale équivalente [5] :

$$
\psi_{\lambda}(x)=\psi_{\lambda}(0)+x \psi_{\lambda}^{\prime}(0)+\int_{0}^{x}(x-t) f_{\lambda}(t) \psi_{\lambda}(t) \mathrm{d} t
$$

avec

$$
f_{\lambda}(x)=-\frac{2 \mu}{\hbar^{2}}\left[E_{\lambda}-U(r)\right]+\frac{\lambda}{r^{2}}
$$

Nous savons d'autre part que $\psi_{\lambda}(x)$ est lié aux fonctions canoniques $\alpha_{\lambda}(x)$ et $\beta_{\lambda}(x)$ par la relation :

$$
\psi_{\lambda}(x)=\psi_{\lambda}(0) \alpha_{\lambda}(x)+\psi_{\lambda}^{\prime}(0) \beta_{\lambda}(x)
$$


et que $\alpha_{\lambda}(x)$ et $\beta_{\lambda}(x)$ sont données par les relations [6] :

$$
\left.\begin{array}{rl}
\alpha_{\lambda}(x) & =\sum_{n=0}^{\infty} A_{n}(x) \\
A_{n}(x) & =\int_{0}^{\dot{x}}(x-t) f_{\lambda}(t) A_{n-1}(t) \mathrm{d} t \\
A_{0}(x) & =1
\end{array}\right\}
$$

et

$$
\left.\begin{array}{l}
\beta_{\lambda}(x)=\sum_{n=0}^{\infty} B_{n}(x) \\
B_{n}(x)=\int_{0}^{x}(x-t) f_{\lambda}(t) B_{n-1}(t) \mathrm{d} t \\
B_{0}(x)=x
\end{array}\right\}
$$

et que les deux séries des fonctions $A_{n}(x)$ et $B_{n}(x)$ sont des séries uniformément et absolument convergentes sans aucune restriction sur la nature du facteur d'énergie $f_{\lambda}(x)[7]$.

2.2 L'énergie de vibration-rotation est usuellement écrite sous la forme [1] :

$$
\begin{aligned}
& E_{v J}=E_{v 0}+J(J+1) B_{v}- {[J(J+1)]^{2} D_{v}+} \\
&+[J(J+1)]^{3} H_{v}+\cdots
\end{aligned}
$$

où $B_{v}, D_{v}, H_{v}, \ldots$ sont les constantes rotationnelles (rapidement décroissantes) du potentiel $U(x)$. Nous écrivons :

$$
E_{\lambda}=\sum_{i=0}^{\infty} \varepsilon_{i} \lambda^{i}
$$

où

$$
\varepsilon_{0}=E_{v 0}, \quad \varepsilon_{1}=B_{v}, \quad \varepsilon_{2}=-D_{v}, \ldots
$$

Le facteur d'énergie s'écrit alors sous la forme :

$$
f_{\lambda}(x)=\sum_{i=0}^{\infty} \mu_{i}(x) \lambda^{i}
$$

avec

$$
\begin{aligned}
& \mu_{0}(x)=-\frac{2 \mu}{\hbar^{2}}\left[\varepsilon_{0}-U(x)\right] \\
& \mu_{1}(x)=-\frac{2 \mu}{\hbar^{2}} \varepsilon_{1}+\frac{1}{\left(r_{\mathrm{e}}+x\right)^{2}} \\
& \mu_{i}(x)=-\frac{2 \mu}{\hbar^{2}} \varepsilon_{i} ; i \geqslant 2 .
\end{aligned}
$$

En remplaçant $f_{\dot{\lambda}}(x)$ par cette valeur dans les fonctions $A_{n}(x)$, on trouve :

$$
\begin{aligned}
& A_{0}(x)=1 \\
& A_{1}(x)=\int_{0}^{x}(x-t) \sum_{i=0}^{\infty} \mu_{i}(t) \lambda^{i} \mathrm{~d} t=\sum_{i=0}^{\infty} a_{i}^{1}(x) \lambda^{i}
\end{aligned}
$$

$$
\begin{aligned}
A_{2}(x)=\int_{0}^{x}(x-t) \sum_{i=0}^{\infty} & \mu_{i}(t) \lambda^{i} \times \\
& \times \sum_{i=0}^{\infty} a_{i}^{1}(t) \lambda^{i} \mathrm{~d} t=\sum_{i=0}^{\infty} a_{i}^{2}(x) \lambda^{i}
\end{aligned}
$$

et finalement :

$$
\alpha_{\lambda}(x)=\sum_{i=0}^{\infty} \mathcal{A}_{i}(x) \lambda^{i} .
$$

On peut obtenir de la même manière :

$$
\beta_{\lambda}(x)=\sum_{i=0}^{\infty} \mathcal{B}_{i}(x) \lambda^{i}
$$

Les fonctions $\mathcal{A}_{i}(x)$ et $\mathcal{B}_{i}(x)$ sont liées simplement aux fonctions $\mu_{i}(x)$ et aux fonctions canoniques de la vibration pure :

$$
\alpha_{0}(x)=\mathcal{A}_{0}(x) \quad \text { et } \quad \beta_{0}(x)=\mathfrak{B}_{0}(x) .
$$

2.3 Les valeurs initiales $\psi_{\lambda}(0)$ et $\psi_{\lambda}^{\prime}(0)$ de la fonction d'onde peuvent être déduites des fonctions canoniques $\alpha_{\lambda}(x)$ et $\beta_{\lambda}(x)$, en se servant des conditions aux limites :

$$
\psi_{\lambda}(x) \underset{\substack{x \rightarrow+\infty \\ x \rightarrow-r_{e}}}{\longrightarrow} 0
$$

En tenant compte des relations (4) et (11), on a :

$$
\frac{\psi_{\lambda}^{\prime}(0)}{\psi_{\lambda}(0)}=-\lim _{x \rightarrow \infty} \frac{\alpha_{\lambda}(x)}{\beta_{\lambda}(x)}
$$

et, en remplaçant $\alpha_{\lambda}(x)$ et $\beta_{\lambda}(x)$ par leurs expressions (9) et (10) :

$$
\frac{\psi_{\lambda}^{\prime}(0)}{\psi_{\lambda}(0)}=\sum_{i=0}^{\infty} l_{i} \lambda^{i}
$$

où les coefficients $l_{i}$ se déduisent simplement des $\mathcal{A}_{i}(\infty)$ et $\mathcal{B}_{i}(\infty)$ [ou de $\mathcal{A}_{i}\left(-r_{\mathrm{e}}\right)$ et $\mathfrak{B}_{i}\left(-r_{\mathrm{e}}\right)$ ].

La fonction d'onde non normalisée devient alors :

$$
\psi_{\lambda}(x)=\psi_{\lambda}(0)\left[\alpha_{\lambda}(x)+\sum_{i=0}^{\infty} l_{i} \lambda^{i} \times \beta_{\lambda}(x)\right]
$$

ou encore en tenant compte de (9) et (10) :

$$
\psi_{\lambda}(x)=\sum_{i=0}^{\infty} \phi_{i}(x) \lambda^{i}
$$

Les fonctions $\phi_{i}(x)(i \geqslant 1)$ seront appelées les harmoniques de rotation de la fonction d'onde $\psi_{\lambda}(x)$.

3. Détermination des harmoniques de rotation. En remplaçant dans l'équation radiale de Schroedin- 
ger la fonction d'onde $\psi_{\lambda}$ par son expression (13), on trouve :

$$
\sum_{i=0}^{\infty} \phi_{i}^{\prime \prime}(x) \lambda^{i}=\sum_{i=0}^{\infty} \mu_{i}(x) \lambda^{i} \times \sum_{i=0}^{\infty} \phi_{i}(x) \lambda^{i}
$$

En tenant compte du fait que cette équation est vérifiée quel que soit $\lambda$, on obtient :

$$
\begin{aligned}
& \phi_{0}^{\prime \prime}-\mu_{0} \phi_{0}=0 \\
& \phi_{1}^{\prime \prime}-\mu_{0} \phi_{1}=\mu_{1} \phi_{0} \\
& \phi_{2}^{\prime \prime}-\mu_{0} \phi_{2}=\mu_{1} \phi_{1}+\mu_{2} \phi_{0} \\
& \cdots \cdots \cdots \cdots \cdots \cdots \cdots \cdots \cdots \cdots \cdots \cdots \cdots \cdots \cdots \cdots \\
& \phi_{i}^{\prime \prime}-\mu_{0} \phi_{i}=\sum_{j=1}^{i} \mu_{j} \phi_{i-j}
\end{aligned}
$$

La $1^{\text {re }}$ de ces équations correspond au cas de la vibration pure $\left[\phi_{0}(x)=\psi_{0}(x) ; J=0\right]$. Les équations suivantes permettent, successivement, de déterminer les harmoniques $\phi_{1}, \phi_{2}, \ldots, \phi_{i}, \ldots$

L'une quelconque de ces équations s'écrit sous la forme :

$$
y_{i}^{\prime \prime}(x)-\mu_{0}(x) y_{i}(x)=s_{i}(x)
$$

avec $s_{0}(x)=0$.

La solution d'une telle équation est [8] :

$$
y_{i}(x)=y_{i}(0) \alpha_{0}(x)+y_{i}^{\prime}(0) \beta_{0}(x)+\sigma_{i}(x)
$$

$\alpha_{0}(x)$ et $\beta_{0}(x)$ étant déduites des expressions (5) et (6) en remplaçant $f_{\lambda}(t)$ par $\mu_{0}(t)$; ce sont les fonctions canoniques de vibration. $\sigma_{i}(x)$ est la solution particulière de l'équation non homogène parfaitement déterminée par les harmoniques d'ordre inférieur à $i$.

Afin de définir les valeurs initiales $y_{i}(0)$ et $y_{i}^{\prime}(0)$ de chaque harmonique, nous nous servons des remarques suivantes :

i) Pour des fonctions d'onde non normalisées $\psi_{\lambda}(x)$ et $\psi_{0}(x)$, on peut toujours prendre - sans nuire à la généralité du problème - :

$$
\psi_{\lambda}(0)=\psi_{0}(0)=1 .
$$

ii) Des conditions aux limites imposées aux fonctions $\psi_{\lambda}(x)$ et $\psi_{0}(x)$,

$$
\left[\psi_{\lambda}(x) \underset{\substack{x \rightarrow \infty \\ x \rightarrow-r_{e}}}{\longrightarrow} 0 ; \quad \psi_{0}(x) \underset{\substack{x \rightarrow-\infty \\ x \rightarrow-r_{e}}}{\longrightarrow} 0\right],
$$

on déduit que ces mêmes conditions s'appliquent à chaque harmonique $\phi_{i}(x)$, sinon les conditions aux limites de $\psi_{\lambda}(x)$ ne seront pas remplies.

Nous déterminons alors les conditions iñitiales de chaque harmonique de la façon suivante :

i) On prend $\phi_{0}(0)=\psi_{0}(0)=1$ et $\phi_{i}(0)=0$ pour $i \neq 0$. Les harmoniques sont alors de la forme : $y_{i}(x)=y_{i}^{\prime}(0) \beta_{0}(x)+\sigma_{i}(x)$. ii) En imposant les conditions aux limites $y_{i}(x) \underset{\substack{x \rightarrow \infty \\ x \rightarrow-r_{e}}}{\longrightarrow} 0$, on trouve :

$$
y_{i}^{\prime}(0)=\lim _{\substack{x \rightarrow \infty \\ x \rightarrow-r_{e}}}-\frac{\sigma_{i}(x)}{\beta_{0}(x)}
$$

qui est parfaitement déterminée par les harmoniques d'ordre inférieur à $i$.

Nous donnons ci-après un exemple des valeurs numériques des harmoniques de certains niveaux de vibration du potentiel de Morse utilisé par Cashion [9]. Ces valeurs sont obtenues en nous servant de l'intégration numérique des équations (14) par la méthode de Runge-Kutta d'ordre 5 , avec un pas $h=0,005 \AA$. Ces valeurs sont comparées à celles de $\psi_{0}(x)$ d'une part et de $\psi_{\lambda}(x)$ d'autre part.

Nous remarquons que, pour une abscisse $x$ donnée, les harmoniques $\phi_{i}(x)$ décroissent rapidement en valeurs absolues quand $i$ augmente.

Tableau I. - Valeurs des harmoniques de rotation

\begin{tabular}{|c|c|c|c|}
\hline & $v=0$ & $v=5$ & $v=10$ \\
\hline $\begin{array}{c}x=0.1 A^{0} \\
\phi_{0} \\
\phi_{1} \\
\phi_{2} \\
-\sum_{i=0} \lambda^{i} \phi_{i} \\
\psi_{\lambda}\end{array}$ & $\begin{array}{l}0.9339664 \\
0.0002736 \\
0.0 \\
--\ldots \\
0.9345137 \\
0.9345142\end{array}$ & 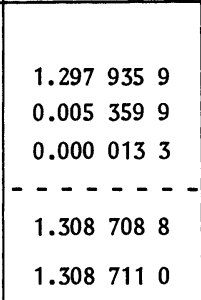 & $\begin{array}{rrrr}-0.143 & 356 & 1 \\
0.003 & 050 & 4 \\
0.000 & 002 & 4 \\
--- & - & - & - \\
-0.137 & 244 & 5 \\
-0.137 & 253 & 2\end{array}$ \\
\hline \begin{tabular}{c}
$x=0.2 A^{\circ}$ \\
$\phi_{0}$ \\
$\phi_{1}$ \\
$\phi_{2}$ \\
\hdashline$\sum_{i=0}^{2} \lambda^{i} \phi_{i}$ \\
$\psi_{\lambda}$
\end{tabular} & $\begin{array}{l}0.6996035 \\
0.000407 \quad 7 \\
0.0 \\
-- \\
0.7004195 \\
0.7004200\end{array}$ & 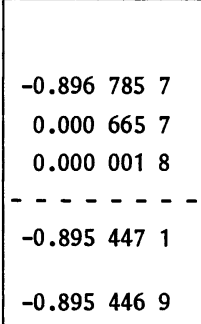 & 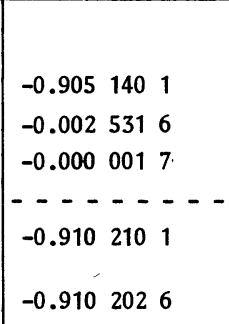 \\
\hline $\begin{array}{c}x=0.3 A^{0} \\
\phi_{0} \\
\phi_{1} \\
\phi_{2} \\
-\sum_{i=0} \lambda^{i} \phi_{i}\end{array}$ & 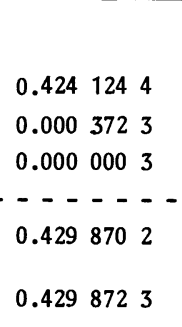 & 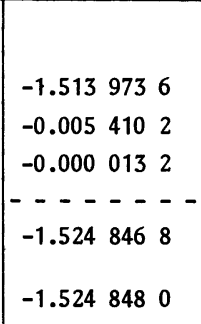 & $\begin{array}{rrrr}0.847 & 575 & 7 \\
-0.001 & 373 & 4 \\
-0.000 & 001 & 9 \\
- & - & - & - \\
0.844 & 821 & 3 \\
0.844824 & 6\end{array}$ \\
\hline
\end{tabular}
$\phi_{i}(x)$ en certains points, pour certains niveaux de vibration $v$ du potentiel de Cashion [9]. La somme des harmoniques est comparée à la fonction d'onde de vibration-rotation $\psi_{\lambda}$ pour $\lambda=J(J+1)=2$.

[Values of the rotational harmonics $\phi_{i}(x)$ at some trial points for different vibrational levels $v$ of the Cashion potential [9]. The sum of the harmonics is compared with the vibration-rotational wavefunction $\psi_{\lambda}$ for $\lambda=J(J+1)=2$.] 
4. Conclusion. - Nous avons démontré que la fonction d'onde de vibration-rotation $\psi_{v J}(x)$ d'une molécule diatomique, s'écrit sous la forme :

$$
\psi_{v J}(x)=\psi_{v 0}(x)+\sum_{i=1}^{\infty} \lambda^{i} \phi_{i}(x) \quad \text { où } \quad \lambda=J(J+1) .
$$

En résumé, et en se servant des notations d'usage, les « harmoniques de rotation » $\phi_{i}(x)$ sont les solutions des équations différentielles non homogènes :

$$
\begin{aligned}
\phi_{1}^{\prime \prime}(x)+\frac{2 \mu}{h^{2}}\left[E_{v}-U(x)\right] \phi_{1}(x) & \\
= & {\left[-\frac{2 \mu}{h^{2}} B_{v}+\frac{1}{r^{2}}\right] \psi_{v 0}(x) }
\end{aligned}
$$

$$
\begin{aligned}
\phi_{2}^{\prime \prime}(x)+\frac{2 \mu}{h^{2}} & {\left[E_{v}-U(x)\right] \phi_{2}(x)=} \\
= & {\left[-\frac{2 \mu}{h^{2}} B_{v}+\frac{1}{r^{2}}\right] \phi_{1}(x)+\frac{2 \mu}{h^{2}} D_{v} \psi_{v 0}(x) }
\end{aligned}
$$

Nous avons déterminé les valeurs initiales des harmoniques $\phi_{i}(x)$ ainsi que leurs conditions aux limites. Une application numérique donnée en exemple montre qu'il suffit de calculer deux ou trois harmoniques pour retrouver la valeur de $\psi_{v J}(x)$ avec une bonne précision.

Les résultats de ce travail ouvrent la voie à une reconsidération utile et élégante des problèmes liés au modèle de l'oscillateur tournant des molécules diatomiques.

\section{Bibliographie}

[1] Herzberg, G., Spectra of Diatomic Molecules, (Van Nostrand, Toronto) 1950.

[2] Rydierg, R., Z. Phys. 73 (1931) 376.

Klein, O., Z. Phys. 73 (1932) 226.

Rees, A. L., Proc. Phys. Soc. 59 (1947) 998.

[3] Kobeissi, H., J. Physique 34 (1973) 235.

Hessel, M. M. et Vidal, C. R., J. Chem. Phys. 70 (1979) 4439.

[4] CoOley, J. W., Math. Comp. 15 (1961) 363.

HajJ, F. Y., Kobeissi, H. et Nassif, N., J. Comput. Phys. 16 (1974) 150.
[5] Kobeissi, H. et Tergiman, S. Y., J. Physique 35 (1974) 635.

[6] Kobeissi, H. et Alameddine, M. A., J. Physique 39 (1978) 43. Kobeissi, H. et DaGHer, M., I.J.Q.C. (Q.C. Symposium 13) (1979) 169.

[7] Alameddine, M. A., Thèse, Lyon (1975).

[8] Hildebrand. F. B.. Introduction to Numerical Anals:sis, (Mc Graw-Hill, New York) 1956.

[9] Cashion, J. K., J. Chem. Phys. 39 (1963) 1872. 